\title{
TV/Series
}

$8 \mid 2015$

Philosopher avec The West Wing

\section{La parrêsia et les rituels de véridiction dans The West Wing}

\section{Valérie Pérez}

\section{(2) OpenEdition}

1 Journals

Édition électronique

URL : http://journals.openedition.org/tvseries/658

DOI : $10.4000 /$ tvseries.658

ISSN : 2266-0909

Éditeur

GRIC - Groupe de recherche Identités et Cultures

Référence électronique

Valérie Pérez, "La parrêsia et les rituels de véridiction dans The West Wing », TV/Series [En ligne], 8 | 2015, mis en ligne le 01 décembre 2015, consulté le 03 mai 2019. URL : http:// journals.openedition.org/tvseries/658; DOI : 10.4000/tvseries.658

Ce document a été généré automatiquement le 3 mai 2019.

\section{(c) (i) (9)}

TV/Series est mis à disposition selon les termes de la licence Creative Commons Attribution - Pas d'Utilisation Commerciale - Pas de Modification 4.0 International. 


\title{
La parrêsia et les rituels de véridiction dans The West Wing
}

\author{
Valérie Pérez
}

Dans la série The West Wing, les spectateurs sont plongés au cœur de la démocratie américaine et de son fonctionnement, sans que l'on puisse voir pour autant les conséquences concrètes sur la vie des Américains, ou à l'étranger, des décisions politiques qui sont prises. Tout est d'abord affaire de discours, de prises de parole; la politique se joue en effet principalement dans le Bureau ovale ou dans la salle de crise (ou même dans l'avion présidentiel «Air Force One ») qui sont des lieux de paroles dont on pourrait dire qu'ils sont ritualisés, en ce qu'ils impliquent d'une part une certaine manière de communiquer et d'autre part un certain rapport à soi-même et aux autres. Ce qui est alors filmé dans l'aile ouest comme une caractéristique de la démocratie, c'est le jeu politique que l'on peut décrire comme le fait de prendre des décisions à l'issue d'échanges verbaux, de débats et de combats, dans lesquels les protagonistes ${ }^{1}$ énoncent et défendent librement une vérité à laquelle ils croient.

2 Ce jeu politique s'accomplit dans le cadre de la Constitution américaine, c'est-à-dire « de quelque chose qui est la loi, qui est tradition, qui est constitution, principe fondamental ${ }^{2}$ .» Qu'il s'agisse d'échanges de points de vue dans un couloir ou de prises de parole plus solennelles en réunion, les actes énonciatifs dans The West Wing sont marqués par la forte conviction des personnages et par leur franc-parler, chacun d'entre eux disant librement la vérité de ce qu'il pense, de ce qu'il pense vraiment être vrai. Il nous semble alors que, sur ce thème du franc-parler, se joue l'un des enjeux majeurs de la série, à savoir la question de la liberté et de la vérité en démocratie, question posée par Michel Foucault à propos de la démocratie athénienne dans ses cours au Collège de France. Ainsi, dans les dialogues qui mettent en scène le président et ses collaborateurs, ou les collaborateurs entre eux, le problème n'est pas de savoir jusqu'où dire la vérité que l'on pense. Mais il semble plutôt que la série expérimente en quoi l'obligation de dire la vérité correspond, en démocratie, à l'exercice risqué de la liberté.

3 Précisons toutefois que tout discours, dans The West Wing, n'est pas discours de vérité. Si l'aile ouest de la Maison-Blanche est, nous semble-t-il, le lieu du parler-vrai, du discours 
politique vrai, l'on peut aussi s'interroger sur la parole qu'entendront les citoyens américains. Ils sont en effet les destinataires d'un discours dont il faut souvent peser et mesurer ce qui pourrait être compris ou mal compris, (comme dans l'épisode 3.8 où se pose le problème de la vache folle et où il faut ménager autant les éleveurs que les consommateurs).

4 À ce sujet, le personnage de C.J. Cregg est particulièrement emblématique puisque, dans les cinq premières saisons, elle fait le lien entre le discours vrai, interne à l'aile ouest, propre au jeu politique, et le discours de communication destiné au grand public, aux citoyens, aux électeurs dont il faut ménager les susceptibilités et les attentes, sans pour autant transmettre de fausses informations. Dans l'épisode 5.18, qui se présente comme un reportage sur l'attachée de presse de la Maison-Blanche, C.J. répète à cinq reprises au moins « je dis la vérité ${ }^{3}$ » et c'est cette exigence du dire-vrai, du franc-parler en tant que principe de vie et principe politique caractéristique de la démocratie qui va nous intéresser.

Sur le plan méthodologique, nous avons déterminé des séquences précises dans les saisons 1,3 et 5 dans lesquelles la parrêsia apparaît comme un dire-vrai irruptif répondant à un problème politique particulier. Mais c'est aussi une notion très large, dont Michel Foucault fait l'historique depuis Platon et Euripide, jusqu'à la spiritualité chrétienne, tout en suggérant des pistes pour la modernité. Nous avons donc choisi les caractéristiques de la parrêsia qui nous permettaient de cerner au mieux les enjeux du franc-parler de la série, à savoir la parrêsia des tragédies d'Euripide, dans lesquelles le dire-vrai peut prendre la forme de l'aveu, celle de l'oracle et celle du discours politique.

\section{Le discours vrai dans The West Wing}

6 The West Wing expérimente différentes formes de discours qui sont autant de procédures et d'opérations par lesquelles la vérité peut être dite. Parmi ces procédures, celle de l'aveu nous paraît particulièrement intéressante parce qu'elle renvoie à la fois à une dimension politique et personnelle. Citons par exemple les anciennes addictions de Leo, la maladie du président, l'assassinat du ministre de la défense du Qumar, Abdul ibn Shareef ${ }^{4}$, etc. Les aveux, écrit Foucault, « sont des actes de parole difficiles, coûteux, péniblement arrachés en dépit de la honte, à travers la vivacité des passions et dans des conditions telles que ce dire-vrai est toujours accompagné de son double d'ombres: mensonges, aveuglements, illusions des personnages ${ }^{5}$. " L'on retrouve bien ici tout ce qui concerne les épisodes sur la maladie du président (deuxième moitié de la saison 2), épisodes dans lesquels Toby joue un rôle de parrèsiaste tout à fait intéressant.

7 Mais l'aveu n'est pas toujours, loin s'en faut, une affaire publique, et la vérité a aussi des rapports plus intimes et plus personnels avec la subjectivité lorsqu'elle prend la forme de la confession. Le 14 épisode de la saison 1 « Take This Sabbath Day » («Observe le jour du sabbat ») s'ouvre sur le refus de la Cour suprême d'exempter de la peine capitale un prévenu condamné pour trafic de drogue et pour le meurtre de deux caïds. Aussitôt se pose la question du dernier recours, puisque «la Constitution habilite le Président à accorder des grâces dans le cas de crimes fédéraux ", comme le rappelle à Sam Seaborn, qui est l'avocat du condamné, l'un de ses anciens camarades de lycée.

Alors à quelles conditions, en démocratie, le président peut-il gracier un condamné, c'està-dire, pour le dire vite, faire passer la morale et les convictions religieuses avant les 
décisions de justice et avant l'opinion majoritaire du peuple? Notre hypothèse est que Bartlet, malgré le fait qu'il n'aime pas la peine de mort, comme il le dit lui-même, malgré ses convictions à l'encontre de toute forme de meurtre (sur le principe qu'il faut punir mais non tuer), ne parvient pas à prendre la décision qui serait salvatrice car il n'a pas croisé, durant cet épisode, la parole vraie qui lui aurait permis d'agir.

Confronté à cette possibilité de la grâce, Bartlet demande pourtant leur avis à toutes les personnes qu'il croise ; Charlie n'aimerait pas voir le meurtrier de sa mère exécuté, mais préfèrerait s'en charger lui-même ; à Joey Lucas, la spécialiste des sondages sourde qu'il vient de rencontrer, Bartlet parle de St-Augustin ${ }^{6}$ et de St-Thomas d'Aquin qui, dit-il, révéraient cette phrase de la Genèse: «qui verse le sang de l'homme verra son sang versé. » Et « Kant, ajoute-t-elle, a dit que la peine de mort est un impératif catégorique ».

Or, malgré le secours de la religion et de la philosophie, Jed Bartlet n'évolue pas et s'abrite derrière sa fonction présidentielle en alléguant que dans ce cas précis « $71 \%$ des Américains seraient pour la peine de mort ». La temporalité de l'épisode suit alors le décompte des heures jusqu'à l'exécution, et chaque personnage entre dans une réflexion personnelle sur le sujet.

11 Dans le Bureau ovale, Bartlet affirme à Leo qu'en graciant le coupable pour la seule raison qu'il n'aime pas la peine de mort, il ouvrirait la voie à toutes sortes de problèmes avec le huitième amendement ${ }^{7}$, si son successeur ne partageait pas son avis. «On ne peut pas exécuter une personne et pas une autre selon l'humeur du Bureau ovale. Cela serait 'cruel et inhabituel'», dit-il, en reprenant les termes mêmes du VIII ${ }^{\mathrm{e}}$ amendement. Dès lors, sa décision est prise, il n'ira pas à l'encontre de l'arrêt de la Cour. À partir de ce moment-là, de cette décision-là, les heures, puis les minutes paraissent précipiter les choses. À 23h57, un prêtre de ses amis rend visite au président. L'exécution est prévue à 00h01.

Bartlet reconnait que tous ses collaborateurs ont cherché des « raisons acceptables aux yeux du public de gracier Simon Cruz ». Mais force est de constater qu'il n'y eut aucun échange de fond sur le problème. Il justifie alors à nouveau sa position par sa fonction. « Le peuple a parlé » dit-il, et il l'a suivi.

13 La peine de mort, en tant que problématique morale et religieuse, n'est pas traitée autrement que dans un cadre juridique. Pourtant, Bartlet avoue au prêtre qu'il a prié pour trouver la sagesse, mais en vain. À la suite de quoi le prêtre lui signifie qu'il n'a pas su voir les signes qui lui était envoyés, qu'il ne s'est pas rendu disponible : «Dieu vous a envoyé un prêtre, un rabbin et une Quaker, M. le président. Sans parler de son fils JésusChrist. Que voulez-vous lui demander de plus?» 'exécution lui est finalement annoncée par un petit papier blanc que C.J. lui remet, dans une mise en scène où le silence et la pénombre accentuent une pesanteur de circonstance. C'est la toute fin de l'épisode.

Le prêtre lui propose d'entendre sa confession, et l'on peut supposer que cette parole de la confession, cette parole vraie (ou du moins que l'on peut supposer telle) laisse entendre le regret, voire le remords de ne pas avoir empêché l'exécution de Simon Cruz. En effet, pour la première fois dans cet épisode, il y a cet autre (le prêtre) qui lui enjoint de parler, de dire vrai sur soi-même ${ }^{8}$. Dans cette affaire, aucun collaborateur du président n'avait été capable d'occuper cette fonction d'interpellateur, qui est celle du parrèsiaste, que Michel Foucault décrit comme un homme capable de voir le vrai et de le dire, un homme intègre moralement, incapable de corruption, et qui, en raison de ses qualités, «pourra exercer, à travers sa parrêsia, l'ascendant qui est nécessaire pour que la cité démocratique soit malgré tout gouvernée ${ }^{9}$; ; malgré tout, c'est-à-dire malgré les obstacles qui peuvent 
surgir au sein même de la démocratie (en l'occurrence, ici, la démocratie américaine autorise la peine de mort).

Par ailleurs, l'épisode joue sur un autre mode du dire-vrai que Foucault étudie pour le comparer à la parrêsia, à savoir le dire-vrai oraculaire. Le prêtre signifie à Bartlet qu'il n'a pas su voir les signes que Dieu lui avait envoyés. Or les dieux, même si on les sollicite, ne sont pas obligés de répondre clairement aux hommes qui les interrogent dans leurs prières. «Au contraire », écrit Foucault, « cela fait partie du dire-vrai oraculaire que la réponse soit telle que les hommes puissent la comprendre ou puissent ne pas la comprendre ${ }^{10}$.»

L'on peut effectivement revoir l'épisode avec cette lecture-là des signes. Chaque personnage y va de sa recherche, y compris le président. Or c'est précisément celui qui avait le pouvoir d'agir qui est resté sourd et immobile. Mais le dire-vrai de l'oracle ne relève pas de la parrêsia. En effet, "le parrèsiaste par définition ne parle pas par énigmes ", écrit Foucault. Il dit au contraire les choses le plus le plus clairement, le plus directement possible, sans aucun déguisement, sans aucun ornement rhétorique, de sorte que les paroles peuvent recevoir immédiatement une valeur prescriptive. Le parrèsiaste ne laisse rien à interpréter. Certes, il laisse quelque chose à faire : il laisse à celui auquel il s'adresse la rude tâche d'avoir le courage d'accepter cette vérité, de la reconnaittre et d'en faire un principe de conduite ${ }^{11}$.

\section{Un dire-vrai offensif}

La question qui se pose donc désormais est de savoir quel type de discours doit être adressé au président pour qu'il puisse gouverner. Il y a à ce sujet une scène qui nous paraît tout à fait intéressante dans l'épisode 1.9, « The short list » (« La liste finale »). Il y est question du choix d'un candidat pour occuper le siège de président à la Cour suprême. Le choix s'est arrêté sur Peyton Cabot Harrison III. Bartlet rencontre Joseph Crouch, le président de la Cour suprême partant à la retraite. Ce dernier critique son choix avec franchise : «Prenez les prochains jours pour accorder à Mendoza l'attention qu'il mérite. [...] Les Américains aiment le cran. Les Républicains en ont. Dans trois ans, l'un d'eux vous battra. » Bartlet lui répond qu'il a beaucoup à gérer : "un Congrès d'opposition, des lobbies très puissants et des médias sans pitié. » « Comme Truman » réplique le juge. «Je ne suis pas Harry Truman » répond Bartlet. "Monsieur Bartlet, rétorque alors le juge, vous n'aviez pas besoin de le préciser ». Le juge sortant met Bartlet en joute et le défie, mais au nom de quoi Joseph Crouch parle-t-il, puisqu'il n'a aucun moyen d'agir?

Dans cette scène, Crouch justifie le fait même de prendre la parole avec franchise et conviction en alléguant son âge et son expérience: «je suis juge de cette Cour depuis trente-huit ans. J'ai commencé l'année de votre entrée à l'université. Je pense avoir gagné le droit de dire mon $\operatorname{mot}^{12} »$. Ce droit de dire son mot, de prendre la parole est un droit politique, mais en même temps il vient de la supériorité de l'âge et de l'expérience. Le ton de Crouch, sévère, autoritaire et condescendant trahit sa colère à l'égard du président, mais cette manière hardie de parler est aussi caractéristique de la parrêsia, en tant que parole vive qui entre dans un jeu agonistique avec celui qui gouverne pour qu'il change sa façon d'agir.

19 Ce que le juge reproche à Bartlet, c'est d'avoir mis Mendoza sur la short list uniquement pour y faire figurer un Hispanique. Et ce reproche le conduit à critiquer une attitude 
frileuse que n'annonçait pas sa campagne électorale: «Vous vouliez tout chambouler pendant la campagne. C'était l'insurrection. Il fallait voir ça. Puis vous avez dévié au centre tout de suite après avoir prêté serment. Au milieu de la route, un endroit tiède, sans risques». Le juge attendait qu'un démocrate soit au pouvoir pour prendre sa retraite : «J'attendais un démocrate, dit-il, et au lieu de cela je vous ai vous ${ }^{13}$ ».

En critiquant le choix de Bartlet, c'est donc toute son action politique qu'il remet en cause. Et de fait, la suite de l'épisode le confirme puisque Mendoza sera finalement choisi. Les faits prouveront d'ailleurs que Harrison était un mauvais choix ${ }^{14}$. Par ailleurs, en évoquant la possibilité de nommer Mendoza, ce n'est pas seulement la question de « comment il faut gouverner » qui est posée que celle de « comment il faut se gouverner soi-même ", d'où cette attaque contre la passivité de Bartlet.

21 L'offensive verbale de Crouch ne relève pas d'un simple avis sur Mendoza - et d'ailleurs, il ne dit rien sur lui, il ne vante ses mérites à aucun moment ${ }^{15}$. Mais il y a dans ces mots adressés au président ce que Foucault appelle le dédoublement de la parrêsia, à savoir que donner un avis pour que la cité soit bien gouvernée est « une activité qui consiste à s'adresser à l'âme de ceux qui doivent gouverner, de manière qu'ils se gouvernent comme il faut et qu'ainsi la cité se trouve gouvernée, elle aussi, comme il faut ${ }^{16}$ ». Il y a donc un " déplacement de la cible ", de la critique du choix du nouveau juge de la Cour suprême à la manière de gouverner le pays et de se gouverner soi pour gouverner les autres.

Crouch remet effectivement en cause l'action politique de Bartlet et Bartlet lui-même et, ce faisant, il lui permet de faire un autre choix, que la série présente comme plus judicieux. À la suite de cela, il est intéressant de se demander quelles sont les conditions, en démocratie, pour que ce genre de prises de parole puisse exister. On voit aussi qu'elles supposent une capacité d'écoute du président, ce qui est assurément l'une de ses qualités, qualité qui relève également de la parrêsia, puisque ce concept renvoie, pour citer encore Foucault, «à cet espèce de pacte, entre celui qui prend le risque de dire la vérité et celui qui accepte de l'entendre ${ }^{17}$ ».

Ainsi, il nous semble que la série The West Wing a pour vocation de montrer que le discours tenu aux gouvernants, pour jouer un rôle en démocratie, doit prendre parfois cette forme offensive, loin de la connivence et de la flatterie. La parrêsia, c'est au contraire la franchise, la liberté de parole, et cette parole, pour citer encore Foucault, est « liée à une organisation sinon exactement législatives, du moins instituée, coutumière, du droit de parole et des privilèges du droit de parole ${ }^{18} »$.

\section{L'ouverture à la parole de l'autre}

Si Joseph Crouch parle au nom de son âge et de son expérience, qu'en est-il des collaborateurs du président ? Comment ont-ils obtenu la parrêsia ? L'on pourrait supposer que le rôle qu'ils ont joué dans l'élection puis la réélection de Bartlet leur a fait acquérir cette légitimité et cette expertise qui autorise à prendre la parole. Mais le problème, c'est que cela reviendrait à considérer la réussite électorale comme une épreuve probatoire, et à réduire le franc-parler à une parole d'expert. Il y a bien sûr dans The West Wing des discours d'expertise, en particulier dans la salle de crise. Mais entre Bartlet et ses proches collaborateurs, les choses se déroulent dans un autre registre. tornade en Oklahoma a fait de nombreux dégâts. Leo veut y envoyer le vice-président. 
C.J., quant à elle, estime que c'est au président Bartlet de s'y rendre, mais le reste de l'équipe ne l'approuve guère. Lors d'une réunion dans le Bureau ovale, d'une façon plutôt amusante, C.J. pince Toby pour cause, dit-elle, de «désaccord professionnel». Ce désaccord est explicité par Léo ${ }^{19}$. Le président demande ensuite à son attachée de presse de dire ce qu'elle pense et celle-ci évoque alors la nécessité de créer une dynamique, dont il pourrait profiter en se rendant en Oklahoma, non pas pour l'image, mais pour porter attention aux sinistrés. Un silence de quelques secondes précède la décision du président qui dit à C.J. : « Vous avez gagné une place à mes côtés dans l'avion ${ }^{20}$ ».

Mais rapidement l'épisode se concentre autour de ce que Leo qualifie de désertion du président ${ }^{21}$, puisqu'en effet Bartlet passe son temps à écouter les rescapés de la tornade et accumule ainsi un retard de plus de vingt-quatre heures dans les affaires à traiter à la Maison-Blanche. L'épisode joue alors sur un va-et-vient entre ces deux lieux, d'une part la Maison-Blanche où l'absence du président crée des incidents et des malentendus, et d'autre part la ville sinistrée dans laquelle Bartlet fait figure de citoyen solidaire venu au secours des autres. Leo McGarry, confronté à des problèmes de plus en plus critiques, somme C.J. d'arranger une situation qu'elle a provoquée. Celle-ci se rend donc auprès du président, qu'elle trouve assis en train de composer une homélie : «Excusez-moi, lui ditelle. Ils nous attendent à Washington". "Après le service", répond Bartlet. "Non, Monsieur le Président. Nous devons y retourner maintenant!, exige C.J., avant d'énumérer les différentes crises en cours. "Les gens ont besoin de moi », rétorque le président en parlant de la ville sinistrée. « Non, Monsieur », réaffirme C.J.

Bartlet est assis, C.J. debout est penchée au dessus de lui. Elle poursuit sur un ton de plus en plus ferme et hardi : « Hier, c'était peut-être le cas, mais aujourd'hui, ils ont besoin de reprendre possession de leur ville et de leur police pour assurer leur sécurité, pas la nôtre! Et de leurs cinquante chambres d'hôtel pour loger les sans-abri. Et ils ont besoin de vous à Washington, afin de créer des emplois et de pouvoir payer les impôts qui serviront à indemniser cette région sinistrée. Que fait-on encore ici, Monsieur? »

Le franc-parler paraît relever ici d'une stratégie de la démonstration. C.J. pèse les causes, les conséquences et anticipe sur l'avenir dans l'intention de persuader Bartlet. De plus, l'interrogation qui clôt cette séquence révèle la manière de parler sans réserve du parrèsiaste (au sens où il n'a pas la réserve du sage, dit Foucault ${ }^{22}$ ). Dans l'avion qui le ramène à Washington, le président convoque $\mathrm{C}$.J. et lui demande si c'est un problème qu'il aille soutenir des gens qui ont besoin d'aide. Il a l'impression d'avoir fait plus de bien en vingt-quatre heures qu'au cours des six derniers mois. Les épisodes précédents ont été marqués par l'enlèvement de Zoey ${ }^{23}$, et C.J. lui avoue qu'elle comprend parfaitement son besoin d'humanité. Alors comment faire pour que le président redevienne le premier des citoyens et gouverne le pays?

Lors de la crise consécutive à l'enlèvement, c'est le XXVe amendement de la Constitution américaine qui a permis de résoudre le problème posé par l'incapacité du président à gouverner ${ }^{24}$. Mais une fois la crise résolue, le président fait montre de faiblesse dans l'exercice du pouvoir. Le problème qui est posé dans cette scène est celui du discours vrai adressé au gouvernant. En demandant à C.J. de s'expliquer ${ }^{25}$, il crée cette situation caractéristique de la parrêsia démocratique que l'on peut définir comme un rapport entre l'obligation de dire la vérité et l'exercice de la liberté. Il s'agit donc, pour poser le problème en termes foucaldiens, d'éprouver comment il est possible de gouverner le prince (donc pour nous ici le président), de telle sorte qu'il se gouverne lui-même et qu'il gouverne les autres. C.J. va tenir au président Bartlet ce que Foucault appelle « un 
discours de la gouvernementalité ${ }^{6}$.» Elle lui dit en effet la chose suivante : «je vous en veux d'avoir fui vos responsabilités. Si vous avez besoin de contact, enseignez les maths dans les ghettos! Les temps ont été durs pour vous. Pour tout le monde. [...] Vous êtes le président des Etats-Unis! Mon président ! J'ai peur. Nous avons tous peur. Le monde est devenu trop dangereux, imprévisible. J'ai besoin que vous soyez de retour. J'ai besoin de vous voir diriger le pays ${ }^{27}$.»

Dans cette séquence, C.J. dit la nécessité, pour le président, de se ressaisir, et en quoi il doit changer (elle joue exactement le même rôle que Leo, il n'est donc pas étonnant qu'elle prenne sa suite dans la saison 6), mais aussi, comme dans le dire-vrai prophétique, elle "dit l'imminence menaçante du lendemain ${ }^{28}$." La scène (le jeu d'acteur) laisse à penser qu'il ne s'agit pas d'un simple argument rhétorique mais de la manifestation d'une urgence. Souvent, dans la série, il est rappelé que Bartlet est le président des États-Unis, c'est-à-dire de la nation la plus puissante du monde, et qu'à ce titre il doit se montrer digne de ses fonctions. En outre, cet énoncé injonctif est en même temps l'affirmation d'une vérité à laquelle elle croit, une vérité qu'elle considère comme authentiquement vraie. « Vous êtes mon président et j'ai peur », lance-t-elle à Bartlet. C'est ce que Foucault appelle un «redoublement de l'énoncé de la vérité par l'énoncé du fait que je pense cette vérité et que, la pensant, je la dis, c'est celle-là qui est indispensable à l'acte parrèsiastique ${ }^{29}$.»

31 C.J. parle en effet avec parrêsia, au sens de Plutarque, à savoir que la parrêsia est " une vertu, une qualité personnelle, un courage ${ }^{30}$. » Ce courage, c'est le courage de la vérité, à savoir que la conseillère du président s'oblige à dire une vérité en laquelle elle croit, en prenant le risque de s'opposer au président, de risquer sa relation avec lui, de le blesser ou de le mettre en colère, ou même pourquoi pas, de perdre son poste. Et c'est précisément ce risque ouvert par le fait de dire la vérité qui fait de la parrêsia « l'exercice le plus haut de la libertés1.»

Une scène de même nature se produit avec Toby, dans laquelle le président lui dit : « Tout autre que moi vous aurait déjà renvoyé, il l'aurait fait depuis longtemps ». En ce sens, la parrêsia est liée à l'organisation politique des États-Unis, en tant que pays dans lequel règne la liberté d'expression, et la possibilité, pour tout citoyen, de dire ce qu'il pense, qui plus est pour ceux des citoyens qui sont au premier rang ${ }^{32}$ dans la sphère du pouvoir. La série joue bien sur ce point de cette circularité repérée par Michel Foucault entre la parrêsia et la démocratie : «Pour qu'il y ait démocratie il faut qu'il ait parrêsia ; pour qu'il y ait parrêsia, il faut qu'il y ait démocratie ${ }^{33}$.»

Bartlet montre également dans cette scène qu'il possède la parrêsia, même s'il ne dit rien. En effet, en contexte démocratique cette notion renvoie à une parole qui laisse sa place à la parole de l'autre. Elle est donc incompatible, en ce sens, avec la tyrannie. Il faut précisément qu'il y ait ces conditions-là de la liberté de parole de tous pour que la parole du parrèsiaste existe et pour qu'elle soit une parole qui persuade. Bartlet, en demandant à C.J. d'expliquer ce qu'elle pense, prend le risque de voir une parole l'emporter sur la sienne. Et il est en effet très intéressant de voir comment la série, à maintes reprises, fait jouer la parrêsia, c'est-à-dire cette parole vraie qui persuade, qui se confronte aux autres paroles et qui l'emporte par la vérité qu'elle énonce.

En tant que caractéristique de la démocratie, la parrêsia renvoie au premier amendement de la Constitution américaine (qui d'ailleurs n'est cité qu'une seule fois par des magiciens qui ont brûlé le drapeau américain à la Maison-Blanche lors de la fête d'anniversaire de Zoey Bartlet en saison 6) - amendement qui porte notamment sur la liberté d'expression ${ }^{34}$ 
. En tant que ressort dramatique, le courage de la vérité relance sans cesse la question de savoir à quelles conditions un homme peut continuer à être un bon président, ou du moins un président acceptable. L'on aurait pu aussi se pencher sur une éventuelle crise de la parrêsia, ou sur ce qui peut la remettre en cause, comme dans la République ${ }^{35}$ où à propos du franc-parler, Platon évoque la cité bariolée et bigarrée dans laquelle il n'y a plus d'unité, puisque chacun suit ses propres décisions. Y-aurait-il aussi un tel risque dans The West Wing?

\section{BIBLIOGRAPHIE}

FOUCAULT Michel, Le gouvernement de soi et des autres, Cours au Collège de France, 1982-1983, Paris, collection Hautes études Gallimard/Seuil, 2008.

FOUCAUlT Michel, Le courage de la vérité, Le gouvernement de soi et des autres II, Paris, Gallimard, Seuil, 2009.

PLATON, La République, Paris, Hachette, 1884. Disponible sur http://gallica.bnf.fr

\section{NOTES}

1. À savoir Jed Bartlet, Josh Lyman, Toby Ziegler, Sam Seaborn, C.J. Cregg et Will Bailey.

2. Michel Foucault, Le gouvernement de soi et des autres, Cours au Collège de France, 1982-1983, Paris, collection Hautes études Gallimard/Seuil, 2008, p. 138.

3. C.J. I tell the truth.

4. À la fin de la saison 3.

5. Foucault [2008], p. 140.

6. Citer un auteur qui a rédigé ses Confessions n'est assurément pas un hasard.

7. « Excessive bail shall not be required, nor excessive fines imposed, nor cruel and unusual punishments inflicted.» («Des cautions excessives ne seront pas exigées, ni des amendes excessives imposées, ni des châtiments cruels et exceptionnels infligés. »)

8. Cet autre, écrit Foucault, « si nécessaire au dire-vrai sur soi même dans la culture chrétienne, où il prend la forme institutionnelle du confesseur ou du directeur de conscience » in [2009], p. 7.

9. Foucault [2008], p. 163-164.

10. Ibid, p. 82.

11. Foucault [2009], p. 17

12. CROUCH. «I've served on this bench for 38 years. I took my seat the year you began college. I believe I've earned the right to say a word. »

13. CROUCH. You ran great guns in the campaign. It was an insurgency, boy, a sight to see. And then you drove to the middle of the road the moment after you took the oath. Just the middle of the road. Nothing but a long line painted yellow.

BARTLET. Excuse me, sir...

CROUCH. I wanted to retire five years ago. But I waited for a Democrat. I wanted a Democrat. $\mathrm{Hmm}$ ! And instead I got you.

14. Il ne tient pas à garantir les droits de la vie privée. 
15. C'est Josh qui fera son portrait élogieux, juste avant que le spectateur le découvre quand il pénètre à la Maison-Blanche : « Mendoza a fait son droit à la dure. Il a été blessé à la jambe, a pris un emploi de bureau, et suivi des cours du soir. Il est brillant, décidé, humain, expérimenté. S'il n'a pas le profil, c'est que tu doutes des Américains.» (Traduction de l'édition française) / "Mendoza went to Law School the hard way. He got shot in the leg, and when they offered him a hundred percent dispensation, he took a desk job instead and went to law school at night. He's brilliant, decisive, compassionate, and experienced. And if you don't think that he's America's idea of a jurist, then you don't have enough faith in Americans. »

16. Foucault [2008], p. 279.

17. Ibid, p. 14.

18. Foucault [2008], p. 275.

19. LEO. She thinks you should go («Elle pense que vous devriez y aller »).

20. Le président est d'ailleurs incapable de justifier son choix : « S’il [le vice-président] découvre pourquoi j'ai accepté, il est plus malin que moi. »

21. "Je crois qu'il vient de déserter " dit-il à Toby Ziegler et à Angela Blake, après que le président lui a raccroché au nez.

22. Foucault [2009], p. 27.

23. La fille cadette du président Bartlet.

24. Bartlet a confié le pouvoir à son concurrent à l'élection présidentielle.

25. BARTLET. I'm asking you a direct question, C.J.

26. Foucault [2008], p. 67.

27. C.J. Honestly, Sir? I resent you ignoring the responsibilities of your office. If you want to reach out and touch people, teach math at East Saint Louis after your term expires.

BARTLET. So you think my staying longer was some sort of personal indulgence?

C.J. I think it's been a difficult time for you sir. For all of us.

BARTLET. What's this really about, C.J.?

C.J. I understand there was no time, sir. I can't imagine what it was like. I don't have children.

BARTLET It's like I knew who I was, then woke up one morning and didn't have a clue.

C.J. I understand, sir. But I need more. You're the President of the United States. My President. I'm frightened. We're all frightened. This is... the world is too dangerous now. Unpredictable. I need you back. I need you to lead. [pause] Is there anything else, Mr. President?

He shakes his head and she gets up and leaves.

28. Foucault [2009], p. 29.

29. Foucault [2008], p. 62.

30. Ibid, p. 68.

31. Ibid., p. 64.

32. À partir de textes d'Euripide, Michel Foucault relève est il y a trois catégories de citoyens. Être au premier rang des citoyens «c'est faire partie de ces quelques-uns qui sont au premier rang de la ville». Dans Les suppliantes, être au premier rang de la ville cela signifie être au premier rang relativement «à la distribution du pouvoir, de l'autorité, de l'influence effective dans la ville ». Il s'agit de la répartition effective « de l'autorité politique, de l'exercice du pouvoir parmi et à l'intérieur de cette masse ou de cet ensemble constitué par les citoyens de droit » (Le gouvernement de soi et des autres, p. 94). Ou encore pour résumer au sujet de ces trois catégories de personnages qui sont trois catégories de citoyens égaux il y a : «les pauvres sans puissance ; et, parmi les puissants, ceux qui se taisent et ne s'occupent pas des affaires de la cité ; et ceux qui se servent et du logos et de la polis » (Ibid., p. 95).

33. Ibid., p. 143.

34. "Congress shall make no law respecting an establishment of religion, or prohibiting the free exercise thereof; or abridging the freedom of speech, or of the press; or the right of the people peaceably to assemble, and to petition the Government for a redress of grievances. » 


\section{RÉSUMÉS}

Le souci de la vérité et le franc-parler font l'objet de mises en scène souvent complexes dans The West Wing. Qu'est-ce qui fonde le pouvoir de parler, si ce n'est pas le statut politique ni le rôle joué par l'individu dans la démocratie ? Par quelles opérations, par quelles procédures, la vérité, d'après The West Wing, peut-elle être dite dans la démocratie américaine?

The desire to speak the truth is often dramatized in complex ways in The West Wing. What gives someone the power to speak if it be not the political status or the role that an individual plays in a democracy? According to the series, by which operations and procedures, should the truth be told within the U.S. democracy?

\section{INDEX}

Mots-clés : À la Maison Blanche, West Wing (The), série télévisée, politique, démocratie, vérité, parole, Foucault Michel, États-Unis

Keywords : West Wing (The), TV series, politics, democracy, truth, speech, Foucault Michel

\section{AUTEUR}

\section{VALÉRIE PÉREZ}

Valérie Pérez enseigne la philosophie à l'ESPÉ de Guadeloupe. Elle est rattachée au laboratoire LLCP de l'université de Paris 8. Elle s'intéresse aux relations entre philosophie et littérature. Ses travaux portent essentiellement sur les auteurs du XVIII e siècle.

Valérie Pérez teaches philosophy at the ESPE in Guadeloupe. She is a member of the LLCP research unit at the University of Paris 8. Her research focuses on the relationships between philosophy and literature, mostly on $18^{\text {th }}$ century authors. 